\title{
Generalization of Poincaré inequality in a Sobolev Space with exponent constant to the case of Sobolev space with a variable exponent
}

\author{
Moulay Rchid Sidi Ammi ${ }^{\mathrm{a}, *}$, Ibrahim Dahib \\ ${ }^{a}$ Department of Mathematics, AMNEA Group, Faculty of Sciences and Techniques, \\ Moulay Ismail University of Meknes, B. P. 509, Errachidia, Morocco. \\ ${ }^{b}$ Department of Mathematics, AMNEA Group, Faculty of Sciences and Techniques, \\ Moulay Ismail University of Meknes, B. P. 509, Errachidia, Morocco.
}

\begin{abstract}
In this work, we study the Poincaré inequality in Sobolev spaces with variable exponent. As a consequence of this result we show the equivalent norms over such cones. The approach we adopt in this work avoids the difficulty arising from the possible lack of density of the space $\mathrm{C}_{0}^{\infty}(\Omega)$.
\end{abstract}

Keywords: Sobolev spaces with variable exponent, Density, Poincaré inequality, Nemytskii operator. 2010 MSC: 46E35, 11R45, 35R45.

\section{Introduction}

In recent years the whole world has undergone various changes in several fields, notably in computing and physics and especially in mechanics, flexible electronics and robotics. As far as we are concerned, we deal with the Poincaré inequality in Sobolev spaces with variable exponent. A multitude of results has emerged in recent years. This growing interest is because many concrete problems can be solved. Sobolev spaces make a special framework of our study, but the problem that we often encounter is that some properties which are verified in a classic Sobolev space but are not verified in a Sobolev space with variable exponent.

The classical Poincaré inequality in Sobolev spaces with a constant exponent asserts that, given any real $p \geqslant 1$, there exists a constant $C=C(\Omega, p)$ such that

$$
\int_{\Omega}|u(x)|^{p} d x \leqslant C \sum_{i=1}^{i=N} \int_{\Omega}\left|\partial_{i} u(x)\right|^{p} d x \quad \text { for all } u \in W_{0}^{1 . p}(\Omega),
$$

where

$$
W_{0}^{1 . p}(\Omega):=\left\{u \in W^{1, p}(\Omega) ; \quad \operatorname{tru}=0 \quad \text { on } \quad \Gamma\right\}
$$

\footnotetext{
*Corresponding author

Email addresses: rachidsidiammi@yahoo.fr (Moulay Rchid Sidi Ammi), i.dahi@edu.umi .ac.ma (Ibrahim Dahi) doi:10.31559/glm2021.10.2.3
} 
Still, this does not provide a satisfactory generalization of the classical Poincare inequality, because the equality which holds for a constant exponent:

$$
\overline{\mathrm{D}}^{\|\cdot\|_{1 \cdot \mathrm{p}}}(\Omega):=\left\{\mathrm{u} \in \mathrm{W}^{1, \mathrm{p}}(\Omega) ; \quad \operatorname{tru}=0 \quad \text { on } \quad \Gamma\right\} .
$$

If we replace $\mathrm{p}$ by $\mathrm{p}(\cdot): \Omega \longrightarrow\left[0, \infty\left[\right.\right.$, then we replace also $\overline{\mathrm{D}}^{\|\cdot\|_{1 \cdot p}}(\Omega)$ by $\overline{\mathrm{D}}^{\|\cdot\|_{1 \cdot p(\cdot)}}(\Omega) \subset\left\{u \in W^{1, p}(\Omega) ; \quad \operatorname{tru}=\right.$ 0 on $\Gamma$. The previous inequality does not correct, see for example [8].

If such a generalized Poincaré inequality can be established for all functions in the space $D(\Omega)$, it cannot be extended in this case by means of a density argument to all functions in the space $W^{1, p}(\Omega)$ whose traces vanish on $\partial \Omega:=\Gamma$.

The purpose of this research note is to now more information about the Poincaré inequality for functions in $\mathrm{D}(\Omega)$ and then extending it by a density argument. We obtain the Poincaré inequality as an immediate corollary of an equivalence of norms in cones of the space $W^{1, p}(\Omega)$ (see Theorems 3.1 and 3.2). There are many questions in this way like does the Poincaré inequality applied when we have space like

$$
\left\{u \in W^{1, p}(\Omega) ; \operatorname{tru}=0 \text { in } \Gamma\right\} .
$$

Thus, avoiding the difficulty arising from the possible lack of density of the space $D(\Omega)$ in this space. secondly, it can be extended at no extra cost to the space

$$
\left\{u \in W^{1, p}(\Omega) ; \quad \operatorname{tru}=0 \quad \text { on } \Gamma_{0}\right\} .
$$

Where $\Gamma_{0} \subset \Gamma$ and we have $\mathrm{d} \Gamma-\operatorname{meas}\left(\Gamma_{0}\right)>0$.

\section{Preliminaries}

Given two normed vector spaces $V$ and $W$, the notation $V \hookrightarrow W$, respectively $V \subset \subset W$, means that $\mathrm{V} \subset \mathrm{W}$ the canonical injection from $\mathrm{V}$ into $\mathrm{W}$ is continuous, respectively compact. The notation $\mathrm{D}(\Omega)$ denotes the space of functions that are infinitely differentiables in $\Omega$ and whose support is a compact subset of $\Omega$.

Given a real number $p \geqslant 1$, the notations $\mathrm{L}^{\mathrm{p}}(\Omega), \mathrm{W}^{1, \mathrm{p}}(\Omega)$ and

$$
\overline{\mathrm{D}}^{\|\cdot\|_{1 \cdot \mathrm{p}}}(\Omega):=\left\{\mathrm{u} \in \mathrm{W}^{1, \mathrm{p}}(\Omega): \quad \operatorname{tru}=0 \quad \text { on } \Gamma\right\},
$$

denote the usual Lebesgue and Sobolev spaces; "usual" means here that the exponent $p \geqslant 1$ is a constant.

Theorem 2.1. (See [6]) Let $\Omega$ be a domain in $\mathbb{R}^{\mathrm{N}}$.

(a) Let $\mathrm{p}(\cdot) \in \mathrm{L}^{\infty}(\Omega)$ where $\mathrm{p}^{-} \geqslant 1$. Then $\left(\mathrm{L}^{\mathrm{p}(\cdot)}(\Omega),\|\cdot\|_{0, \mathrm{p}(\cdot)}\right)$ is a separable Banach space, where the norm is defined as follows

$$
\|v\|_{0, p(\cdot)}:=\inf \left\{\lambda>0 ; \int_{\Omega}\left|\frac{v(x)}{\lambda}\right|^{p(x)} d x \leqslant 1\right\} .
$$

Moreover, if $\mathrm{p}^{-}>1$, the space $\mathrm{L}^{\mathrm{p}(\cdot)}(\Omega)$ is uniformly convex, hence reflexive.

(b) let $\mathrm{p}_{1}(\cdot) \in \mathrm{L}^{\infty}(\Omega)$ and $\mathrm{p}_{2}(\cdot) \in \mathrm{L}^{\infty}(\Omega)$ where $\mathrm{p}_{1}^{-} \geqslant 1$ and $\mathrm{p}_{2}^{-} \geqslant 1$. Then

$$
\mathrm{L}^{\mathrm{p}_{2}(\cdot)}(\Omega) \hookrightarrow \mathrm{L}^{\mathrm{p}_{1}(\cdot)}(\Omega),
$$

if and only if

$$
p_{1}(x) \leqslant p_{2}(x) \quad \text { for all } x \in \Omega .
$$

(c) Given the function $\mathrm{p}(\cdot) \in \mathrm{L}^{\infty}(\Omega)$ such that $\mathrm{p}^{-}>1$, let $\mathrm{p}^{\prime}(\cdot) \in \mathrm{L}^{\infty}(\Omega)$ defined by

$$
\frac{1}{p(x)}+\frac{1}{p^{\prime}(x)}=1 \quad x \in \Omega \text {. }
$$


Then, for any function $\mathrm{u} \in \mathrm{L}^{\mathrm{p}^{\prime}(\cdot)}(\Omega)$, the following linear functional:

$$
\begin{aligned}
\mathrm{L}^{\mathrm{p}(\cdot)}(\Omega) & \longrightarrow \mathbb{R} \\
v & \longrightarrow \int_{\Omega} \mathrm{u}(\mathrm{x}) \boldsymbol{v}(\mathrm{x}) \mathrm{d} x .
\end{aligned}
$$

is continuous. Conversely, given any continuous linear functional $\mathrm{L}^{\mathrm{p}(\cdot)}(\Omega) \longrightarrow \mathbb{R}$, there exists a unique function $\mathfrak{u}_{\ell} \in \mathrm{L}^{\mathrm{p}^{\prime}(\cdot)}(\Omega)$ such that

$$
\begin{aligned}
\mathrm{L}^{\mathrm{p}(\cdot)}(\Omega) & \longrightarrow \mathbb{R} \\
v & \longrightarrow \int_{\Omega} \mathrm{u}_{\ell}(\mathrm{x}) \boldsymbol{v}(\mathrm{x}) \mathrm{d} \mathrm{x} .
\end{aligned}
$$

Given a function $p(\cdot) \in \mathrm{L}^{\infty}(\Omega)$, that satisfies $\mathrm{p}^{-} \geqslant 1$, the Sobolev space $\mathrm{W}^{1, p}(\Omega)$

$$
W^{1, p(\cdot)}(\Omega)=\left\{u \in L^{p(\cdot)}(\Omega) ; \partial_{i} u \in L^{p(\cdot)}(\Omega), 1 \leqslant k \leqslant N\right\},
$$

where, for each $1 \leqslant k \leqslant N$, $\partial_{i}$ denotes the distributional derivative operator with respect to the $i$-the variable.

Theorem 2.2. (See [7]) Let $\Omega$ a domain in $\mathbb{R}^{\mathrm{N}}$.

(a) Let $\mathrm{p}(\cdot) \in \mathrm{L}^{\infty}(\Omega)$ such that $\mathrm{p}^{-} \geqslant 1$, then the space $\mathrm{W}^{1, \mathrm{p}(\cdot)}(\Omega)$ Equipped with the norm

$$
\begin{aligned}
W^{1, p(\cdot)}(\Omega) & \longrightarrow \mathbb{R} \\
v & \longrightarrow\|v\|_{1, p(\cdot)}=\|v\|_{0, p(\cdot)}+\sum_{i=1}^{i=N}\left\|\partial_{i} v\right\|_{0, p(\cdot)}
\end{aligned}
$$

is a Banach space. If $\mathrm{p}^{-}>1$, hence the space $\mathrm{W}^{1, \mathrm{p}(\cdot)}(\Omega)$ is reflexive.

(b) Let suppose that $\mathrm{p}_{1}(\cdot) \in \mathrm{L}^{\infty}(\Omega)$ with $\mathrm{p}_{1}^{-} \geqslant 1$ and $\mathrm{p}_{2}(\cdot) \in \mathrm{L}^{\infty}(\Omega)$ with $\mathrm{p}_{2}^{-} \geqslant 1$ such that

$$
p_{2}(x) \leqslant p_{1}(x) \quad \text { for all } x \in \Omega \text {. }
$$

Then

$$
W^{1, p_{2}(\cdot)}(\Omega) \hookrightarrow W^{1, p_{1}(\cdot)}(\Omega) .
$$

(c) Let $\mathrm{p}(\cdot) \in \bar{\Omega}$ such that $\mathrm{p}^{-} \geqslant 1$. For each element $\mathrm{x} \in \bar{\Omega}$, we define

$$
p^{\partial}(x):=\left\{\begin{array}{cc}
\frac{N p(x)}{N-p(x)}, & \text { si } p(x)<N \\
\infty & \text { si } p(x) \geqslant N .
\end{array}\right.
$$

Suppose that there exists a function $\mathrm{q}(\cdot) \in \mathrm{C}(\bar{\Omega})$ satisfying

$$
1 \leqslant q(x)<p^{\partial}(x) \text { pour tout } x \in \bar{\Omega} .
$$

Then the following injection:

$$
\mathrm{W}^{1, \mathrm{p}(\cdot)}(\Omega) \hookrightarrow \hookrightarrow \mathrm{L}^{\mathrm{q}(\cdot)}(\Omega),
$$

is compact. Thus, in particular, the trace operator

$$
\mathrm{W}^{1, p(\cdot)}(\Omega) \hookrightarrow \hookrightarrow \mathrm{L}^{\mathrm{p}(\cdot)}(\Omega) .
$$

Finally, we state several results and properties of traces of functions in the space $\mathrm{W}^{1, p(\cdot)}(\Omega)$ 


\section{Poincaré Inequality in a Cone in $W^{1, p(\cdot)}(\Omega)$}

We now establish some results extended for the usual Sobolev spaces to Sobolev spaces with variable exponents established by Jebelean and Precup [5].

Recall that, as a domain in $R^{N}$, the open set $\Omega$ is in particular connected. So, a function $u \in W^{1,1}(\Omega)$ that satisfies $\partial_{i} u=0$ a.e. in $\Omega, 1 \leqslant i \leqslant N$, is a constant function.

Theorem 3.1. Let $\Omega \subset \mathbb{R}^{\mathrm{N}}$. Let $\mathrm{p}(\cdot) \in \mathrm{C}(\bar{\Omega})$ such that $\mathrm{p}^{-}>1$ and we suppose that $\mathrm{U} \neq\{0\} \subset \mathrm{W}^{1, p(\cdot)}(\Omega)$ a cone that is sequentially weakly closed and that does not contain nonzero constant functions. Then there exists a constant $\mathrm{C}=\mathrm{C}(\mathrm{U})$ such that

$$
\|\mathrm{u}\|_{1, \mathrm{p}(\cdot)} \leqslant \mathrm{C}|\mathrm{u}|_{1, \mathrm{p}(\cdot),}, \quad \forall \mathrm{u} \in \mathrm{u},
$$

where the semi-norm is defined as follows $|\cdot|_{1, p(\cdot)}: W^{1, p(\cdot)}(\Omega) \longrightarrow \mathbb{R}$,

$$
\mathrm{u} \longrightarrow|\mathrm{u}|_{1, p(\cdot)}
$$

with $|u|_{1, p(\cdot)}=\sum_{i=1}^{N}\left\|\partial_{i} u\right\|_{0, p(\cdot)}$.

Idea of the proof

We use absurd reasoning to prove inequality.

Theorem 3.2. We suppose that the hypothesis of the above theorem about $\mathrm{p}(\cdot), \Omega$ and a cone $\mathrm{U}$ are satisfied and let

$$
\mu(\mathrm{U}):=\inf \left\{|w|_{1, p(\cdot)} ; \forall w \in \mathrm{U}, \quad\|w\|_{0, p(\cdot)}=1\right\},
$$

then $\mu>0$ and there exists $v \in \mathrm{U}$ such that

$$
\|w\|_{0, p(\cdot)}=1 \text { et } \mu(\mathrm{U})=|w|_{1, p(\cdot)} .
$$

Hence

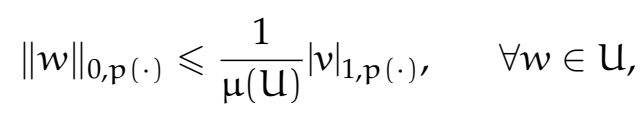

where $\frac{1}{\mu(\mathrm{U})}$ is the best possible constant in this Poincaré inequality.

Idea of the proof

We use the previous theorem, and we prove that $C$ is the best possible constant in this Poincaré inequality.

\section{Applications}

Our first application is applied to the case where the cone $U$ in $W^{1, p(\cdot)}(\Omega)$ is a subspace, associated with an homogeneous Dirichlet condition.

Theorem 4.1. Assume that $\Omega$ is a domain of $\mathbb{R}^{\mathrm{N}}$, and let $\Gamma_{0}$ a subset of $\Gamma=\partial \Omega$ with meas $\left(\Gamma_{0}\right)>0$. Let $\mathrm{p}(\cdot) \in \mathrm{C}(\bar{\Omega})$ such that $\mathrm{p}^{-}>1$

and thus

$$
\mathrm{U}:=\left\{v \in \mathrm{W}^{1, \mathrm{p}(\cdot)}(\Omega), \quad \operatorname{trv}=0 \quad \text { on } \quad \Gamma_{0}\right\},
$$

where the trace operator $\operatorname{tr}$ is defined as follows

$$
\operatorname{trv}: W^{1, p(\cdot)}(\Omega) \longrightarrow L^{p(\cdot)}(\Gamma)
$$

Then there exists a constant $\mathrm{C}=\mathrm{C}(\mathrm{U})$ such that

$$
\|v\|_{0, \mathrm{p}(\cdot)} \leqslant \mathrm{C}|v|_{1, \mathrm{p}(\cdot)}, \quad \forall v \in \mathrm{U} .
$$




\section{Idea of the proof}

1. It suffices to verify that the above set $U$ satisfies the assumptions of Theorem 3.1;

2. To show that $U$ is sequentially weakly closed in $W^{1, p(\cdot)}(\Omega)$;

3. It remains to show that $|v|_{1, p(\cdot)}$ is a norm over the space $\mathrm{U}$.

Therefore its trace on $\Gamma$ is a constant function that takes the same value, and this value is zero since the trace vanishes on $\Gamma_{0}$ and $\mathrm{d} \Gamma-\operatorname{meas}\left(\Gamma_{0}\right)>0$.

When $\Gamma=\Gamma_{0}$, the above theorem shows that the Poincaré inequality holds. Then, there exists a constant C $>0$ such that

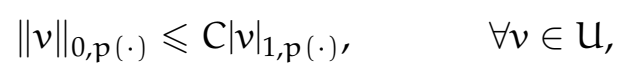

where

$$
\mathrm{U}=\mathrm{W}^{1, \mathrm{p}(\cdot)}(\Omega):=\left\{v \in \mathrm{W}^{1, \mathrm{p}(\cdot)}(\Omega), \quad \operatorname{tr} v=0 \quad \text { on } \quad \Gamma=\Gamma_{0}\right\} .
$$

But, as indicated in the introduction, the space

$$
\overline{\mathrm{D}}^{\|\cdot\|_{1 \cdot p}}(\Omega)=\mathrm{W}_{0}^{1 \cdot p}(\Omega),
$$

may be only strictly contained in the space. Hence in this case, establishing the Poincare inequality for all functions in $D(\Omega)$ would yield the same inequality for all functions in $W^{1 . p}(\Omega)$, but not for all functions in $\mathrm{W}^{1, p(\cdot)}(\Omega)$.

Remark 4.2. If $\mathrm{p}(\cdot) \in \mathrm{L}^{\infty}(\Omega)$ and $\mathrm{p}^{-} \geqslant 1$ satisfies the following Diening-Fan-Zhao-Zhikov condition: there exists a constant $C$ such that

$$
\begin{gathered}
|p(x)-p(y)| \leqslant \frac{C}{|\operatorname{Ln}| x-y \|^{\prime}} \\
\forall x, y \in \bar{\Omega} \text { verifying }\|x-y\|<\frac{1}{2},
\end{gathered}
$$

where $\|$.$\| is the Euclidean norm in \mathbb{R}^{\mathrm{N}}$. Then the spaces $W_{0}^{1, p(\cdot)}(\Omega)$ and $W_{0}^{1, p(\cdot)}(\Omega)$ coincide.

Our second application is to "genuine" cones $\mathrm{U}$ (i.e., which are not subspaces) of a specific form.

Theorem 4.3. Let $\Omega$ a subset of $\mathbb{R}^{\mathrm{N}}$. Let $\mathrm{p}(\cdot) \in \mathrm{C}(\bar{\Omega})$ and $\mathrm{q}(\cdot) \in \mathrm{C}(\bar{\Omega})$ with $\mathrm{q}^{-}>1$ and such that

$$
\mathrm{q}(\mathrm{x})<\mathrm{p}^{\mathrm{\partial}}(\mathrm{x}), \quad \forall \mathrm{x} \in \bar{\Omega}
$$

where the function $\mathrm{p}^{\mathrm{\partial}}: \bar{\Omega} \longrightarrow \mathbb{R}$ is defined as follows

$$
p^{\partial}(x)= \begin{cases}\frac{N p(x)}{N-p(x)}, & \text { if } p(x)<N \\ \infty & \text { if } p(x) \geqslant N\end{cases}
$$

and $\mathrm{q}_{1}(\cdot) \in \mathrm{C}(\bar{\Omega})$ is defined by

$$
\frac{1}{\mathrm{q}(\mathrm{x})}+\frac{1}{\mathrm{q}_{1}(\mathrm{x})}=1, \quad \forall x \in \bar{\Omega} .
$$

Let $\mathrm{f}: \Omega \times \mathbb{R} \longrightarrow \mathbb{R}$ be a Carathéodory function such that, for all $\lambda \geqslant 0$ there exists two constants $\mathrm{C}_{\lambda}^{-}$and $\mathrm{C}_{\lambda}^{+}$ with the property that

$$
C_{\lambda}^{-} f(x, s) \leqslant f(x, \lambda s) \leqslant C_{\lambda}^{+} f(x, s), \quad \text { for all } x \in \Omega \text { and all } s \in \mathbb{R} .
$$

that there exist a non-negative function $\mathrm{a} \in \mathrm{L}^{\mathrm{q}^{\prime}(\cdot)}(\Omega)$ and a positive constant $\mathrm{b}$ such that

$$
|f(x, s)| \leqslant a(x)+b|s|^{q(x)-1} \quad \text { for all } x \in \Omega \text { and all } s \in \mathbb{R} .
$$


Finally, assume that the set

$$
\mathrm{U}:=\left\{w \in W^{1, p(\cdot)}(\Omega) ; \quad \int_{\Omega} f(x, w(x)) d x=0\right\},
$$

does not contain nonzero constant functions.

Then there exists a constant $\mathrm{C}=\mathrm{C}(\mathrm{U})$ such that

$$
\|w\|_{1, \mathrm{p}(\cdot)} \leqslant \mathrm{C}|w|_{1, \mathrm{p}(\cdot)}, \quad \forall w \in \mathrm{U} .
$$

Idea of the proof.

1. Verify that the above set $\mathrm{U}$ satisfies the assumption of Theorem 3.1.

2. Verify that $\mathrm{U}$ is sequentially weakly closed. By using this inequality

$$
|f(x, s)| \leqslant a(x)+b|s|^{\frac{q(x)}{q_{1}(x)}} \text { for almost all } x \in \Omega \text { and all } s \in \mathbb{R} \text {, }
$$

the associated Nemytskii operator $\mathrm{N}_{f}$, defined for any function $w \in \mathrm{L}^{\mathrm{q}(\cdot)}(\Omega)$

$$
\mathrm{N}_{\mathrm{f}} \mathcal{w}(x)=f(x, w(x)), \quad \forall x \in \Omega .
$$

maps $L^{q(\cdot)}(\Omega)$ into $L^{q_{1}(\cdot)}(\Omega)$, and is continuous.

The mapping

$$
w \in L^{q(\cdot)}(\Omega) \longrightarrow \int_{\Omega} f(x, w(x)) d x
$$

is thus continuous, as composed of the continuous mappings

$$
\begin{aligned}
\mathrm{L}^{\mathrm{q}(\cdot)}(\Omega) & \stackrel{\mathrm{N}_{\mathrm{f}}}{\longrightarrow} \mathrm{L}^{\mathrm{q}_{1}(\cdot)}(\Omega) \stackrel{\Phi}{\rightarrow} \mathbb{R} \\
v & \longrightarrow \mathrm{N}_{\mathrm{f}} w \longrightarrow \int_{\Omega} \mathrm{f}(x, w(x)) \mathrm{d} x
\end{aligned}
$$

On another hand, if $w_{k}$ converges to $w$ as $k$ converges to $+\infty$ in $L^{q(\cdot)}(\Omega)$, then

$$
\begin{aligned}
\phi \circ N_{f}\left(w_{k}\right) & \longrightarrow \phi \circ N_{f}(w), \\
\int_{\Omega} f\left(x, w_{k}(x)\right) d x & \longrightarrow \int_{\Omega} f(x, w(x)) d x, \\
k & \longrightarrow \infty
\end{aligned}
$$

Since $w_{k} \in U$, we have $\int_{\Omega} f\left(x, w_{k}(x)\right) d x=0$. Then,

$$
\int_{\Omega} f(x, w(x)) d x=\lim _{k \rightarrow+\infty} \int_{\Omega} f\left(x, w_{k}(x)\right) d x=0 .
$$

Hence

$$
\int_{\Omega} f(x, w(x)) d x=0
$$

Finally $w \in \mathrm{U}$. Then, if applying Theorem 3.1, there exists we get $\mathrm{C}=\mathrm{C}(\mathrm{U})>0$ such that

$$
\|w\|_{1, p(\cdot)} \leqslant \mathrm{C}|w|_{1, \mathrm{p}(\cdot)}, \quad \forall w \in \mathrm{U} .
$$


Remark 4.4. An example of a function satisfies the assumptions of Theorem 4.2 is given by

$$
\begin{aligned}
f: \Omega \times \mathbb{R} & \longrightarrow \mathbb{R} \\
\quad(x, s) & \longrightarrow f(x, s)=|s|^{q(x)-2} s .
\end{aligned}
$$

If $\lambda \in[0,1]$, then there exists constants $C_{\lambda}^{-}$and $C_{\lambda}^{+}$for each $\lambda \geqslant 0$ such that

$$
\lambda^{q^{+}} \leqslant \lambda^{q(x)} \leqslant \lambda^{q^{-}} .
$$

If $\lambda \in[1,+\infty[$, we have

$$
\lambda^{q^{-}} \leqslant \lambda^{q(x)} \leqslant \lambda^{q^{+}},
$$

where $q^{+}=\sup _{x \in \bar{\Omega}}|q(x)|$ and $|f(x, s)|=|s|^{q(x)-1}$ for almost all $x \in \Omega$ and all $s \in \mathbb{R}$, for a constant $p$.

Remark 4.5. Theorem 2.1(c) shows that the same conclusion holds if the set $\mathrm{U}$ is of the more general form

$$
\mathrm{U}:=\left\{v \in \mathrm{W}^{1, p(\cdot)}(\Omega) ; \quad \int_{\Omega} h(x) f(x, v(x)) d x=0\right\},
$$

where $h$ is a given non-negative function in the space $L^{q(\cdot)}(\Omega)$.

\section{Conclusion and perspectives}

This work has many advantages, but the most important thing is to put this result into practice and to broaden our knowledge. Our goal is to find the Poincare inequality in the Sobolev spaces of the weighted variable exponent and the resolution of certain systems using certain methods in these enlarged spaces.

\section{Acknowledgment}

The authors are thankful for the referee for any critical remarks, suggestions and comments about this work.

\section{References}

[1] D. E. Edmunds and J. Rázkosnik, Sobolev embedding with variable exponent, Studia Mathematics 143 (2000), 267-293.

[2] D.E. Edmunds, J. Rázkosnik, Sobolev embedding with variable exponent, II, Math. Nachr. 246-247 (2002), 53-67.

[3] Fan, X. and Zhao, D., On the spaces $L^{p(x)}(\Omega)$ and $W^{m, p(x)}(\Omega)$, J. Math. Anal. Appl., 263, 2001, 424-446.

[4] H. Brezis, Functional analysis, Sobolev spaces and partial differential equations, Springer-Verlag (2010).

[5] Jebelean, P. and Precup.R, Poincaré inequalities in reflexive cones, Appl. Math. Letters, 24, 2011, $359-363$. https:/ / doi.org/10.1016/j.aml.2010.10.024

[6] Kovacik, O. and Rakosnik, J., On spaces $\mathrm{L}^{\mathrm{p}(\mathrm{x})}$ and $\mathrm{W}^{\mathrm{k}, \mathrm{p}(\mathrm{x})}$, Czechoslovak. Math. J., 41, 1991, 592-618.

[7] L. Diening, P. Harjulehto, P. Hasto, and M. Ruzicka. Lebesgue and Sobolev spaces with variable exponents. Springer, 2011.

[8] Maeda, F.Y., Poincare type inequalities for variable exponents. J. Inequalities Pure Appl. Math., 9, 2008, Article 68, 1-5.

[9] O.A. Ladyzenskaia, N.N. Ural'tzeva, Linear and Quasi-linear Equations, Academic Press, New York, 1988.

[10] Philippe. G, George,A Poincaré Inequality in a Sobolev Space with a Variable Exponent. Springer Verlag, 2011. 